\title{
DGLAP and BFKL equations in $N=4$ SUSY
}

\author{
L. N. Lipatov * \\ Petersburg Nuclear Physics Institute \\ Gatchina, 188300, St. Petersburg - Russia
}

\begin{abstract}
The properties of the BFKL kernel in the next-to-leading approximation in QCD and in supersymmetric models are discussed. The maximal transcendality of anomalous dimensions in $N=4 \mathrm{SUSY}$ is formulated. The explicit expressions for the anomalous dimensions up to four loops are given. Their asymptotic behavior at $j \rightarrow \infty$ and in the singular points $j=1,0,-1, \ldots$ is compared with predictions.
\end{abstract}

\section{Introduction}

The QCD scattering amplitude in the leading logarithmic approximation (LLA) has the Regge-type asymptotics with the gluon trajectory in one loop given below

$$
\omega\left(-|q|^{2}\right)=-\frac{\alpha_{c}}{4 \pi^{2}} N_{c} \int d^{2} k \frac{|q|^{2}}{|k|^{2}|q-k|^{2}} \approx-\frac{\alpha_{c}}{2 \pi} \ln \frac{\left|q^{2}\right|}{\lambda^{2}} .
$$

In the coordinate representation the Balitsky-Fadin-Kuraev-Lipatov (BFKL) equation for the Pomeron wave function can be written as follows [2]

$$
E \Psi\left(\vec{\rho}_{1}, \vec{\rho}_{2}\right)=H_{12} \Psi\left(\vec{\rho}_{1}, \vec{\rho}_{2}\right), \Delta=-\frac{\alpha_{s} N_{c}}{2 \pi} \min E,
$$

where $\Delta$ is the Pomeron intercept. The BFKL Hamiltonian in the operator form is simple [3]

$$
H_{12}=\ln \left|p_{1} p_{2}\right|^{2}+\frac{1}{p_{1} p_{2}^{*}} \ln \left|\rho_{12}\right|^{2} p_{1} p_{2}^{*}+\frac{1}{p_{1}^{*} p_{2}} \ln \left|\rho_{12}\right|^{2} p_{1}^{*} p_{2}-4 \psi(1),
$$

where $\rho_{12}=\rho_{1}-\rho_{2}$. It is invariant under the Möbius transformation [4] and has the property of the holomorphic separability [3]. The quantum numbers of the Möbius group are the anomalous dimension $\gamma=\frac{1}{2}+i \nu$ and the conformal spins $n$.

The Bartels-Kwiecinskii-Praszalowicz (BKP) equation [5] for the $n$-gluon composite states in the large- $N_{c}$ limit has the duality symmetry [6], is integrable $[3,7]$ and equivalent to a Scgrödinger equation for the Heisenberg spin model [8]. To restore the $s$-channel unitarity one can use the effective field theory for Reggeized gluons [9]-[11].

\section{DGLAP and BFKL dynamics in $N=4$ SUSY}

In the next-to-leading approximation the eigenvalue of the BFKL kernel is written below

$$
\omega=\omega_{0}(n, \gamma)+4 \hat{a}^{2} \Delta(n, \gamma), \hat{a}=g^{2} N_{c} /\left(16 \pi^{2}\right) .
$$

In QCD $\Delta(n, \gamma)$ is a non-analytic function of the conformal spin $|n|[12,13]$, but in $N=4$ SUSY the Kroniker symbols are cancelled [13]. In this model we obtain for $\Delta(n, \gamma)$ the result

$$
\Delta(n, \gamma)=\phi(M)+\phi\left(M^{*}\right)-\frac{\rho(M)+\rho\left(M^{*}\right)}{2 \hat{a} / \omega}, M=\gamma+\frac{|n|}{2},
$$

${ }^{*}$ Marie Curie Excellence Chair 


$$
\rho(M)=\beta^{\prime}(M)+\frac{1}{2} \zeta(2), \beta^{\prime}(z)=\frac{1}{4}\left[\Psi^{\prime}\left(\frac{z+1}{2}\right)-\Psi^{\prime}\left(\frac{z}{2}\right)\right],
$$

where all special functions have the maximal trancedentality property [13]

$$
\phi(M)=3 \zeta(3)+\Psi^{\prime \prime}(M)-2 \Phi(M)+2 \beta^{\prime}(M)(\Psi(1)-\Psi(M))
$$

and

$$
\Phi(M)=\sum_{k=0}^{\infty} \frac{\beta^{\prime}(k+1)}{k+M}+\sum_{k=0}^{\infty} \frac{(-1)^{k}}{k+M}\left(\Psi^{\prime}(k+1)-\frac{\Psi(k+1)-\Psi(1)}{k+M}\right) .
$$

For one loop anomalous dimension matrix in the case $N=4$ the calculations were performed in Ref. [14]. In this model all twist-2 operators belong to the same supermultiplet and have the following anomalous dimension

$$
\gamma_{u n i}^{(0)}(j)=-4 S_{1}(j-2), S_{r}(j)=\sum_{i=1}^{j} \frac{1}{i^{r}} .
$$

Note, that this function has the maximal transcedentality. It leads to an integrability of evolution equations for matrix elements of quasi-partonic operators in $N=4$ SUSY [14].

\section{Two and three loop results}

Using maximal transcedentality hypothesis [13] and QCD results [15], one can calculate also the anomalous dimensions in two and three loops $[16,17]$

$$
\gamma_{u n i}(j)=\hat{a} \gamma_{u n i}^{(0)}(j)+\hat{a}^{2} \gamma_{u n i}^{(1)}(j)+\hat{a}^{3} \gamma_{u n i}^{(2)}(j)+\ldots,
$$

where

$$
\frac{1}{8} \gamma_{u n i}^{(1)}(j+2)=2 S_{1}(j)\left(S_{2}(j)+S_{-2}(j)\right)-2 S_{-2,1}(j)+S_{3}(j)+S_{-3}(j)
$$

and

$$
\begin{gathered}
\frac{1}{32} \gamma_{u n i}^{(2)}(j+2)=24 S_{-2,1,1,1}-12\left(S_{-3,1,1}+S_{-2,1,2}+S_{-2,2,1}\right)+6\left(S_{-4,1}+S_{-3,2}+S_{-2,3}\right) \\
-3 S_{-5}-2 S_{3} S_{-2}-S_{5}-2 S_{1}^{2}\left(3 S_{-3}+S_{3}-2 S_{-2,1}\right)-S_{2}\left(S_{-3}+S_{3}-2 S_{-2,1}\right) \\
-S_{1}\left(8 \bar{S}_{-4}+\bar{S}_{-2}^{2}+4 S_{2} \bar{S}_{-2}+2 S_{2}^{2}\right)-S_{1}\left(3 S_{4}-12 \bar{S}_{-3,1}-10 \bar{S}_{-2,2}+16 \bar{S}_{-2,1,1}\right)
\end{gathered}
$$

Here the corresponding harmonic sums are defined below

$$
\begin{gathered}
S_{a}(j)=\sum_{m=1}^{j} \frac{1}{m^{a}}, S_{a, b, c, \cdots}(j)=\sum_{m=1}^{j} \frac{1}{m^{a}} S_{b, c, \cdots}(m), \\
S_{-a}(j)=\sum_{m=1}^{j} \frac{(-1)^{m}}{m^{a}}, S_{-a, b, \cdots}(j)=\sum_{m=1}^{j} \frac{(-1)^{m}}{m^{a}} S_{b, \cdots}(m) .
\end{gathered}
$$




\section{$4 \quad$ Weak and strong coupling regimes}

The above results are in an agreement with the BFKL prediction [13] for the singularities at $j \rightarrow 1$

$$
\gamma_{u n i}^{N=4}(j)=\hat{a} \frac{4}{\omega}-32 \zeta_{3} \hat{a}^{2}+32 \zeta_{3} \hat{a}^{3} \frac{1}{\omega}-\frac{16 \hat{a}^{4}}{\omega^{4}}\left(32 \zeta_{3}+\frac{\pi^{4}}{9} \omega\right) .
$$

Note, that recently the four-loop result $\gamma_{u n i}^{(3)}(j)$ was calculated with the use of the asymptotic Bethe ansatz [18]. It turned out, that the obtained expression has the singularity in $j=1$ incompatible with the BFKL prediction. A simple modification of the four loop result taking into account the wrapping effect gives an agreement with the BFKL equation and the following non-linear equation for $j+2 r=\omega \rightarrow 0(r=0,1,2, \ldots)$

$$
\omega \gamma_{u n i}=\gamma_{u n i}^{2}+16 \hat{a}^{2}\left(S_{2}+\zeta_{2}-S_{1}^{2}\right)+4 \hat{a}\left(1-\omega S_{1}-\omega^{2}\left(S_{2}+\zeta_{2}\right)+\gamma^{2}\left(S_{2}+S_{-2}\right)\right)
$$

generalizing the resummation of the double logarithmic terms $\sim \alpha / \omega^{2}$ (cf. [19]).

Further, the universal anomalous dimension at large $j$

$$
\gamma_{u n i}^{N=4}=a(z) \ln j, z=\frac{\alpha N_{c}}{\pi}=4 \hat{a}
$$

can be found from our results up to three loops

$$
a(z)=-z+\frac{\pi^{2}}{12} z^{2}-\frac{11}{720} \pi^{4} z^{3}+\ldots
$$

It is remarkable, that using the AdS/CFT correspondence [20] between the superstring model on the anti-de-Sitter space and the $N=4$ supersymmetric Yang-Mills theory A. Polyakov with collaborators calculated the coefficient $a(z)$ in the strong coupling limit [21]

$$
\lim _{z \rightarrow \infty} a(z)=-z^{1 / 2}+\frac{3 \ln 2}{4 \pi}+\ldots
$$

In Ref. [16] the resummation of the perturbative expansion of $a(z)$ was suggested, which reproduces approximately the three-loop result and the strong coupling limit.

The perturbative calculations of the anomalous dimension at large $j$ are in agreement with the recent papers $[22,23]$, where integral equations was derived from the integrability of the model. One can rewrite the Eden-Staudacher integral equation [22] as a set of linear equations [24]

$$
\begin{gathered}
a_{n, \epsilon}=\sum_{n^{\prime}=1}^{\infty} K_{n, n^{\prime}}(\epsilon)\left(\delta_{n^{\prime}, 1}-a_{n^{\prime}, \epsilon}\right), K_{n, n^{\prime}}(\epsilon)= \\
2 n \sum_{R=0}^{\infty}(-1)^{R} \frac{2^{-2 R-n-n^{\prime}}}{\epsilon^{2 R+n+n^{\prime}}} \zeta\left(2 R+n+n^{\prime}\right) \frac{\left(2 R+n+n^{\prime}-1\right) !\left(2 R+n+n^{\prime}\right) !}{R !(R+n) !\left(R+n^{\prime}\right) !\left(R+n+n^{\prime}\right) !},
\end{gathered}
$$

where the function $a(z)$ is expressed in terms of $a_{1, \epsilon}$

$$
a(z)=\frac{2\left(1-a_{1, \epsilon}\right)}{\epsilon^{2}}, \epsilon=\frac{1}{g \sqrt{2}} .
$$


We can easiliy prove, that the maximal transcedentality property for $a(z)$ is valid in all orders of the perturbation theory and the coefficients in front of the products of the corresponding $\zeta$ functions are integer numbers [24]. It is possibly to show [24], that the asymptotic behaviour of $a(z)$ in the case of the Beisert-Eden-Staudacher equation [22] in the agreement with the AdS/CFT prediction [21].

$$
\lim _{g \rightarrow \infty} \gamma_{\operatorname{sing}}=\frac{2}{\epsilon} \frac{I_{1}\left(2 \epsilon^{-1}\right)}{I_{0}\left(2 \epsilon^{-1}\right)} \approx 2 \sqrt{2} g-\frac{1}{2}
$$

Note, that the intercept of the BFKL Pomeron in the strong coupling limit was calculated in Refs. [17] and [25]

\section{References}

[1] Slides: http://indico. cern. ch/contributionDisplay . py? contribId=188\&sessionId=8\&conf Id=9499

[2] V.S. Fadin, E.A. Kuraev, L.N. Lipatov, Phys. Lett. B60 50 (1975); Ya.Ya. Balitsky, L.N. Lipatov, Yad. Fiz. 281597 (1978).

[3] L.N. Lipatov, Phys.Lett. B309 394 (1993).

[4] L.N. Lipatov, JETP 901536 (1986).

[5] J. Bartels, Nucl.Phys. B175 365 (1980); J. Kwiecinski, M. Praszalowicz, Phys.Lett.B B94 413 (1980).

[6] L.N. Lipatov, Nucl.Phys. B548 328 (1999).

[7] L.N. Lipatov High energy asymptotics of multi-colour QCD and exactly solvable lattice models, Padova preprint DFPD/93/TH/70, hep-th/9311037, unpublished.

[8] L.N. Lipatov, JETP Lett. 59596 (1994);

L.D. Faddeev, G.P. Korchemsky, Phys. Lett. B342 311 (1995).

[9] L.N. Lipatov, Nucl.Phys. B365 614 (1991).

[10] L.N. Lipatov, Nucl.Phys. B452 369 (1995).

[11] E. Antonov, I. Cherednikov, E. Kuraev, L. Lipatov, Nucl.Phys. B721 111 (2005).

[12] V. Fadin, L. Lipatov, Phys. Lett. B429 127 (1998); M. Ciafaloni, G. Camici, Phys. Lett. B430 349 (1998).

[13] A. Kotikov, L. Lipatov, Nucl.Phys. B582 19 (2000), Nucl.Phys. B661 19 (2003).

[14] L. Lipatov, talk at "Perspectives in Hadronic Physics", Proc. of Conf. ICTP. Triest, Italy, May 1997.

[15] S. Moch, J.A.M. Vermaseren, A. Vogt, Nucl.Phys. B688 101 (2004).

[16] A. Kotikov, L. Lipatov, V. Velizhanin, Phys. Lett. B557 114 (2003).

[17] A. Kotikov, L. Lipatov, A. Onishchenko, V. Velizhanin, Phys. Lett. B595 521 (2004); Phys. Lett. B632 754 (2006).

[18] A.V. Kotikov, L.N. Lipatov, A. Rej, M. Staudacher, V.N. Velizhanion, arXiv:hep-th/0704.3586v.

[19] R. Kirschner, L.N. Lipatov, Phys.Rev. D26 1202 (1982.)

[20] J.M. Maldacena, Adv. Theor. Math. Phys. 2231 (1998).

[21] S.S. Gubser, I.R. Klebanov, A.M. Polyakov, Nucl.Phys. B636 99 (2002).

[22] B. Eden, M. Staudacher, J. Stat. Mech. 0611 P014 (2006).

[23] N. Beisert, B. Eden, M. Staudacher, J. Stat. Mech. 0701 P021 (2007).

[24] A.V. Kotikov, L.N. Lipatov, Nucl. Phys. B769 217 (2007).

[25] R.C. Brower, J. Polchinski, M.J. Strassler, C-I Tan, preprint hep-th/0603115. 